\title{
Código de buena práctica para el control del dolor oncológico
}

\author{
J. R. González-Escalada ${ }^{1}$, A. Camba ${ }^{2}$, A. Casas $^{3}$, P. Gascón ${ }^{4}$, I. Herruzo ${ }^{5}$, J. M. Núñez-Olarte 6 ,
} A. Ramos-Aguerri ${ }^{7}$, J. Trelis ${ }^{8}$ y L. M. Torres ${ }^{9}$

${ }^{1}$ Unidad del Dolor. Hospital Ramón y Cajal. Madrid. ${ }^{2}$ Servicio de Anestesiología-Reanimación y Tratamiento del Dolor. C.H.A. Marcide. Ferrol. Presidente de la SED. ${ }^{3}$ Área Sectorial de Oncología Médica. Sevilla. ${ }^{4}$ Servicio de Oncología Médica. Hospital Clínic. Barcelona. ${ }^{5}$ Servicio de Oncología Radioterápica. Hospital Carlos Haya. Málaga. Presidente Asesor de SEOR. ${ }^{6}$ Unidad de Cuidados Paliativos. Hospital General Universitario Gregorio Marañón. Madrid. ${ }^{7}$ Servicio de Oncología Radioterápica. Hospital Ramón y Cajal. Madrid. ${ }^{8}$ Servicio de Soporte Integral y Cuidados Paliativos. Instituto Catalán de Oncología. Hospital Germans Trias i Pujol. Badalona, Barcelona. Vicepresidente SECPAL. ${ }^{9}$ Servicio de Anestesia-Reanimación y Tratamiento del Dolor. Hospital Universitario Puerta del Mar. Cádiz.

González-Escalada JR, Camba A, Casas A, Gascón P, Herruzo I, Núñez-Olarte JM, Ramos-Aguerri A, Trelis J, Torres LM. Código de buena práctica para el control del dolor oncológico. Rev Soc Esp Dolor 2011; 2: 98-117. medical possibilities is common among patients, and it is our duty to help them become aware tha medicine offers a number of therapies that actually can suppress pain and relieve suffering. This awareness is key to increase trust in therapy and improve doctor-patient relationships.

Facing this challenge SECPAL (Sociedad Española de Cuidados Paliativos), SED (Sociedad Española del Dolor), SEOM (Sociedad Española de Oncología Médica), and SEOR (Sociedad Española de Oncología Radioterápica) unite in a joint venture to create ALIADO (Alianza Contra el Dolor Oncológico), an interdisciplinary work team emerging with the goal of raising awareness and involvement among health providers regarding the need for quality of life improvement in cancer patients with pain.

In this respect, ALIADO's first initiative is the development of a good practice code for the management of cancer pain in an attempt to contribute to the further understanding and management of these patients.

(C) 2011 Sociedad Española del Dolor: Published by Arán Ediciones, S.L.

Key word: Cancer pain. Breakthrough pain. Cancer. Opioids. Fentanyl.

\section{RESUMEN}

En muchas ocasiones, los pacientes con cáncer no expresan su dolor por miedo a que el médico desvíe su atención hacia este síntoma, en lugar de centrarse en el trata- 
miento de su enfermedad principal. No obstante, el paciente debe saber que el tratamiento de su dolor no solo no interfiere con la terapia curativa, sino que colabora con ella. Entre los enfermos, es habitual el temor a que el avance de la enfermedad les lleve a situaciones de sufrimiento insostenible que supere todas las posibilidades médicas, pero es nuestro deber ayudarles a ser conscientes de que la medicina ofrece un abanico de terapias capaces de aliviar su dolor y evitar su sufrimiento. Este conocimiento es esencial para aumentar su confianza en el tratamiento y mejorar la relación médico-paciente.

Frente a este desafío, SECPAL (Sociedad Española de Cuidados Paliativos), SED (Sociedad Española del Dolor), SEOM (Sociedad Española de Oncología Médica) y SEOR (Sociedad Española de Oncología Radioterápica) unen sus esfuerzos y crean ALIADO (Alianza Contra el Dolor Oncológico), un grupo de trabajo interdisciplinario que nace con el objetivo de concienciar e implicar a todo el colectivo médico en la necesidad de mejorar la calidad de vida del paciente con dolor oncológico.

La primera iniciativa de ALIADO en este sentido es la elaboración del Código de buena praxis para el tratamiento del dolor oncológico, que pretende contribuir al avance en el conocimiento y el manejo de estos pacientes.

(C) 2011 Sociedad Española del Dolor. Publicado por Arán Ediciones, S.L.

Palabras clave: Dolor oncológico. Dolor irruptivo. Cáncer. Opioides. Fentanilo.

\section{INTRODUCCIÓN}

Los médicos que tratamos al paciente con cáncer, estamos preocupados por su dolor.

Muchas veces los pacientes no expresan adecuadamente su dolor por miedo a que el médico desvíe su atención hacia el síntoma en lugar de centrarse en el tratamiento de su enfermedad principal. El primer tratamiento del dolor debido al cáncer es el propio tratamiento oncológico que al curar o reducir el tumor alivia también el dolor. Es muy importante que el paciente sepa que los profesionales estamos dispuestos a ayudarle en todos los aspectos físicos y espirituales, atendiendo de forma integral su proceso y que el hecho de atender y tratar los síntomas entra dentro del planteamiento de tratamiento global de la enfermedad. Es esencial que el paciente sometido a terapia para el cán- cer sea conocedor de que el tratamiento de su dolor no solo no interfiere con la terapia curativa sino que ciertamente colabora con ella. Este conocimiento es esencial porque aumenta la confianza en el tratamiento y mejora la relación médico-paciente.

En ocasiones los enfermos temen que el avance de la enfermedad les lleve a situaciones de sufrimiento insostenible que supere todas las posibilidades médicas. Es importante que sean conscientes que la medicina posee suficientes remedios farmacológicos para combatir el dolor y que existen otros procedimientos capaces de aliviarles y evitar su sufrimiento en caso necesario.

Siempre se debe investigar el origen del dolor en el paciente oncológico, ya que en ocasiones puede no estar relacionado con el cáncer.

Cuando aparece dolor en un paciente diagnosticado de cáncer, es un error frecuente asumir que siempre es producto de la enfermedad. Nunca debería afirmarse esto sin una investigación suficiente. Este axioma contiene una doble vertiente: la primera se establece sobre la realidad de que es posible padecer cáncer sin dolor, por lo que es necesario desvincular ambas circunstancias. De hecho, algunas patologías oncológicas son indoloras en sí mismas y solo la aparición de complicaciones son causa de dolor; como también ocurre en otras enfermedades no relacionadas con el cáncer. Muchos tumores solo producen dolor en las últimas fases de su desarrollo o en las últimas semanas de la enfermedad. Según la bibliografía revisada, el dolor es un síntoma frecuente en el paciente oncológico pero muestra su mayor incidencia en las fases evolucionadas de la enfermedad; casi todos los autores (1-11) estiman que se presenta entre el 70 y el $90 \%$ de los pacientes en fases avanzadas o terminales. Esta afirmación pone de relieve la importancia del tratamiento correcto de estos pacientes, pero no excluye que muchos enfermos con cáncer estén libres de dolor en los periodos iniciales o durante la evolución temprana de la enfermedad. De hecho, muchos de los autores establecen que esta incidencia varía entre el 30 y el $50 \%$ de los pacientes en fase de tratamiento oncológico dependiendo del tipo de cáncer $(6,7,10)$, lo que quiere decir que al menos la mitad de los pacientes están libres de dolor en este periodo.

El dolor producido por una neoplasia puede ser debido a un efecto directo de la enfermedad o bien debido a los tratamientos empleados para curarla. Pero esto no es distinto a lo que ocurre con otras patologías no oncológicas causantes de dolor crónico. A pesar de ello, los textos que se ocupan del dolor en el paciente oncológico olvidan incluir en sus clasificaciones el grupo de pacientes que sufren dolor no relacionado 
con el cáncer (1-6,8-10). Es muy demostrativo que existen muchos estudios epidemiológicos que ofrecen cifras de incidencia de dolor crónico no oncológico en la población y solo encontramos referencias anecdóticas y no verdaderos estudios epidemiológicos, que estimen la incidencia real de dolor crónico no oncológico en la población que sufre o ha sufrido cáncer (12).

La segunda verdad es que es posible que un paciente con cáncer sufra otro proceso que se acompañe de dolor o bien que su estado anímico amplifique la percepción dolorosa de tal forma que una leve molestia, relacionada o no con su enfermedad, se eleve a la categoría de dolor intolerable por su implicación negativa en las sospechas evolutivas.

En un estudio reciente se evaluaron 24 PET de pacientes con cáncer. En 21 se encontraron captaciones de FDG en las extremidades. De ellos, 14 tenían sintomatología de dolor en los miembros y el diagnóstico final fue de osteoartritis (artrosis), bursitis y artropatías diversas (13). Así pues, mayoritariamente el dolor de estos pacientes estaba ligado a enfermedades crónicas no oncológicas. Este ejemplo ayuda a cambiar el concepto de que el dolor y la evolución del cáncer van siempre de la mano y que su aumento o presencia siempre auguran un empeoramiento de la enfermedad de consecuencias catastróficas.

En los pacientes con cáncer es frecuente la coexistencia de depresión $(14,15)$. La incidencia de depresión mayor afecta al 5-26\% de los enfermos (15). La coexistencia de depresión y síntomas físicos en los pacientes con cáncer ha sido evaluada en estudios recientes $(16,17)$ en los que se establece que el $83 \%$ de los pacientes oncológicos que están deprimidos presentan dolor (16), y por tanto, se hace necesario distinguir entre lo que es una mera somatización depresiva de un dolor somático (17). No es de extrañar el buen resultado que aportan muchas estrategias elaboradas sobre conceptos de apoyo conductual $(18,19)$ con clara repercusión en la mejoría de la calidad de vida de estos pacientes.

El médico tiene por tanto el deber y la obligación de investigar el origen del dolor antes de iniciar un tratamiento analgésico, ya que de su correcta evaluación dependerá el éxito o fracaso del control sintomático del paciente durante su evolución posterior. No en vano, todas las guías clínicas coinciden en que antes de iniciar el tratamiento analgésico se debe realizar una correcta evaluación del cuadro clínico, del estado anímico y físico, y de las circunstancias que rodean al paciente $(1-3,5,6,8-11)$. No cabe duda que en el diagnóstico diferencial de este acto habrá que considerar siempre la posibilidad de que el dolor del paciente tenga un origen no relacionado con el cáncer o su tratamiento; el solo conocimiento de ello por el paciente puede disminuir la percepción dolorosa, siendo esta estrategia más eficaz que cualquier analgésico. Entendemos que este axioma no establece competencia alguna con la aseveración de que el dolor y el sufrimiento del paciente con cáncer deberán ser aliviados siempre.

Tras su diagnóstico y evaluación, el dolor oncológico tiene tratamiento y se puede controlar.

El dolor condiciona todo el entorno vital del paciente y además es el síntoma más frecuente en el cáncer ya que se estipula que ocurre en una cuarta parte de los pacientes en el momento del diagnóstico, en un tercio de los que están siendo sometidos a tratamiento y en tres cuartas partes de los pacientes con enfermedad avanzada, por lo que dada la alta incidencia del cáncer en nuestro país, que aproximadamente afecta a 400 pacientes por cada 100.000 habitantes y año, el dolor oncológico constituye un importante problema socio sanitario al que es obligatorio dar respuesta.

Además es el síntoma más temido. Su presencia repercute en el confort del paciente, afecta gravemente a todas sus actividades, motivaciones, bloquea las interacciones con su familia y amigos y destruye la calidad de vida y cualquier percepción de felicidad. En nuestro país el dolor por cáncer no tiene una atención multidisciplinar, como sería lo óptimo y deseable según establecen todas las guías (20), por lo que más de la mitad de los pacientes que son atendidos en el departamento de oncología experimentan dolor y la mayoría de los pacientes y sus médicos creen que el dolor no está adecuadamente atendido (21).

La importancia de aliviar el dolor y la amplia disponibilidad de terapias efectivas, como la radioterapia en esquemas hipofraccionados, que bien indicada proporciona un alivio rápido y duradero del dolor con un bajo coste (22), y los tratamientos farmacológicos bien estructurados y aplicados, hace imperativo que los médicos y los demás profesionales implicados, especialmente atención primaria, paliativistas y enfermería, ayuden al paciente a controlar su dolor adecuadamente.

Ello implica familiarizarse con su patogenia, conocer las técnicas básicas de evaluación del dolor, vencer las barreras comunes al uso de narcóticos, conocer y administrar eficazmente las diferentes alternativas farmacológicas, derivar cuando sea necesario para la práctica de técnicas anestésicas o neuroquirúrgicas y conocer técnicas de apoyo psicológico encaminadas al alivio del dolor asociado al cáncer.

Consideramos de gran utilidad una guía publicada por la National Comprehensive Cancer Network 
(NCCN) en 2009 (23) que tiene en cuenta no solo la evaluación inicial del dolor, sino su reevaluación periódica adaptándose a la respuesta terapéutica y otras circunstancias del paciente, valorando el riesgo del infratratamiento y atendiendo al soporte psicológico y social, además de tener en cuenta aspectos de educación del paciente, la familia y/o el cuidador.

Un perfil importante que no se debe descuidar en el enfoque y manejo de estos pacientes, es el recoger los aspectos relacionados con el tratamiento de los efectos secundarios provocados por las propias terapias analgésicas, sobre todo los opiáceos y los coadyuvantes. También deben ser consideradas otras terapias alternativas como la hipnosis, los masajes, la musicoterapia, las técnicas de relajación, la acupuntura..., que se han mostrado útiles en el control del dolor o la ansiedad relacionada, así como atender los aspectos relacionados con las creencias y las necesidades espirituales (24).

Actualmente se dispone de suficientes recursos farmacológicos como para asegurar el control del dolor en más del $95 \%$ de los pacientes. Existen analgésicos opioides y no opioides que se pueden adaptar a la graduación de la intensidad del dolor oncológico en cada situación. En este sentido, la escala analgésica del dolor, promovida por la OMS, sigue siendo una estrategia válida para el tratamiento del dolor oncológico y su uso se debe seguir promoviendo en todos los niveles asistenciales (25).

La administración sanitaria tiene el deber de proporcionar recursos y accesibilidad equitativa a los tratamientos especializados de todos los enfermos con dolor por cáncer.

Los pacientes con dolor oncológico son usuarios de cualquier recurso de la red sanitaria, desde los hospitales, a centros de atención primaria y desde centros socio sanitarios asistidos a centros residenciales. Por este motivo, se hace necesario implementar la formación de los profesionales involucrados en el manejo de estos pacientes así como aplicar otras medidas encaminadas a fomentar políticas sanitarias que permitan ofrecer accesibilidad equitativa y universal a los recursos específicos.

La formación de profesionales ha sido asumida por las sociedades científicas con programas de formación continuada específicos y algunas sociedades como la Sociedad Española de Oncología Médica (SEOM) (26), la Sociedad Española de Oncología Radioterápica (SEOR) (27), la Sociedad Española de Cuidados Paliativos (SECPAL) (28) y la Sociedad Española de Medicina Familiar y Comunitaria (SEMFYC) (29), han creado grupos de expertos en el tema con el resultado de crear manuales o guías de práctica clínica.
El otro grupo de medidas están englobadas dentro del apartado de políticas sanitarias. Entre ellas destacan las relacionadas con los cuidados paliativos, desde las recomendaciones del Consejo de Europa (30) hasta la aprobación de la Estrategia en Cuidados Paliativos del Sistema Nacional de Salud (31). Estas estrategias permiten desarrollar de forma homogénea la creación y evaluación de equipos de cuidados paliativos en el territorio nacional.

\section{IMPACTO DEL DOLOR PARA EL PACIENTE}

Cuando a una persona se le comunica que padece un cáncer, asocia casi automáticamente la enfermedad con la idea de sufrir dolor. ¿Qué significa el dolor para el paciente oncológico? Algunas de las primeras frases que con frecuencia escuchamos en la consulta tras informar del diagnóstico, son preguntas o afirmaciones relacionadas con el dolor y formuladas por el paciente o sus familiares. “¿Tendrá muchos dolores?”..., “queremos que no sufra”..., “ise podrá controlar el dolor?”; son frases que expresan miedo ante el sufrimiento insuperable, estrechamente relacionado con el diagnóstico de cáncer.

En el enfermo oncológico, el temor a padecer dolor genera sensación de ansiedad y de incertidumbre ante el sufrimiento, de desasosiego ante la aparición de un posible síntoma que se desconoce en cualidad e intensidad pero que se da como cierto $(32,33)$. El miedo a sufrir puede ser en ocasiones casi tan intenso como el temor a perder la propia vida (34).

Una ayuda valiosa para superar todas estas incógnitas es mejorar el conocimiento sobre el dolor con una información específica y de calidad $(35,36)$.

El dolor oncológico produce un gran impacto en la vida de los pacientes con cáncer, que se manifiesta por la disminución de su capacidad funcional (ECOG, Karnofsky) y la aparición de síntomas físicos, afectivos y emocionales que alteran sustancialmente la calidad de vida. Como consecuencia de todo ello se producen diversas alteraciones y manifestaciones clínicas como la sensación de cansancio permanente, disminución de la capacidad de trabajo, percepción de minusvalía para desplazarse, falta de interés por el entorno y sentimientos de aislamiento del mundo, dando todo ello lugar a la pérdida de la interacción familiar y social (37).

Conocer mejor las causas y los factores que giran en torno al dolor oncológico así como los tratamientos disponibles, puede ayudar a combatirlo de una forma más efectiva. Algunos de los aspectos básicos que se deben conocer son los siguientes: 
- El dolor oncológico se puede aliviar: gracias a los tratamientos actualmente disponibles, el dolor oncológico se puede controlar en más del $95 \%$ de los casos.

- Muchas personas temen tomar opioides potentes por miedo a convertirse en "adictos". Sin embargo, muchos estudios han demostrado que los pacientes oncológicos que toman morfina $\mathrm{u}$ otros opioides para el dolor no se acostumbran a ellos ni se hacen "adictos". Es necesario tranquilizar a los pacientes, disipar estas dudas e insistir en que deben tomar la medicación para el dolor cuando realmente hace falta, sobre todo utilizar el medicamento adecuado cuando el dolor se presenta y no cuando la situación es insostenible y no puede aguantar más, desterrando el miedo a que el medicamento no haga efecto en el futuro por acostumbramiento (38). Hay que dar a conocer la escasa tolerancia que se desarrolla durante los tratamientos prolongados con opioides en otros cuadros de dolor crónico de muy larga evolución (39).

- Existe una amplia gama de tratamientos y de técnicas para controlar el dolor oncológico. Siempre se debe confiar en su existencia ya que aseguran que siempre se puede hacer algo más para controlar el dolor por intenso que este sea (24).

Por lo general, un buen control del dolor suele ser el resultado de un cuidadoso plan que tiene en cuenta las medicaciones que son efectivas y las que no y que ajusta las dosis en función de las características de cada circunstancia y cada paciente. Pacientes y profesionales deben trabajar siempre unidos para lograr un buen plan analgésico, individualizado y que reúna un buen manejo profesional con una buena comunicación médico-paciente $(40,41)$.

Todo paciente oncológico tiene derecho a recibir un adecuado tratamiento del dolor $(34,42)$. Es necesario que el profesional establezca una buena comunicación con el paciente y gane su confianza, transmitiendo la seguridad de que el oncólogo tratará adecuadamente el dolor cuando se presente o le enviara a otro especialista en caso necesario (43).

\section{EL DOLOR ONCOLÓGICO ES UN PROBLEMA DE SALUD PÚBLICA}

El dolor oncológico sigue representando un problema de salud pública en nuestro país, a pesar de los grandes avances producidos en los últimos 15 años en nuestra capacidad para aliviarlo. Son mayoría los enfermos de cáncer que sufren por dolor en el curso de su enfermedad.
En todo el conjunto del estado español se produjeron 100.244 defunciones por cáncer en el año 2004 (31). Aplicando las cifras de prevalencia conocidas de dolor oncológico en las fases iniciales (41\%) y avanzadas de la enfermedad cancerosa (75\%) (44), se puede concluir sin temor a equivocarnos que, cada año, al menos 75.000 españoles se enfrentan al sufrimiento que provoca el dolor por cáncer.

Para muchos pacientes, el dolor es la consecuencia más temida de la enfermedad cancerosa. El dolor no aliviado produce un sufrimiento innecesario, y puede resultar psicológicamente devastador para el paciente. La consecuencia puede ser el agotamiento físico y mental, junto con la pérdida de la esperanza y el deseo de vivir (44).

Existe evidencia científica acumulada de la presencia de niveles significativamente superiores de depresión y ansiedad en los pacientes cancerosos con dolor, respecto de aquellos que no lo tienen. La capacidad del dolor canceroso para generar depresión y ansiedad no parece tener relación con cuadros depresivos previos (44).

Por otro lado, las proyecciones generadas por instituciones como la Organización Mundial de la Salud (OMS) señalan que en las próximas décadas asistiremos a un incremento mundial en el número de muertes anuales por cáncer, así como en mayores de 65 años. Con el envejecimiento progresivo de la población podremos situarnos en escenarios en los que el ratio de familiares cuidadores por paciente se aproxime a $2: 1$ (45).

El desarrollo en las últimas dos décadas de Estrategias Nacionales y Regionales que han priorizado la atención a los enfermos que padecen dolor por cáncer, han resultado claves para comprender las mejoras en la atención que están percibiendo nuestros pacientes y sus familias (31). El actual clima social apoya la implicación de los profesionales sanitarios, agrupados en sus respectivas sociedades científicas (SED, SEOM, SEOR, SECPAL etc.), en la consecución de objetivos de calidad mensurables tales como: adecuación de la normativa de prescripción de opioides, accesibilidad de los mismos para su uso como analgésicos en el dolor canceroso, incrementos en el consumo sanitario etc. (45).

Podemos estar razonablemente satisfechos de los avances en este campo en nuestro país en los últimos 20 años. No obstante, la magnitud del problema, junto con el impacto que produce el dolor oncológico en el paciente y en su entorno familiar, siguen estimulando a los profesionales sanitarios implicados en su manejo a buscar fórmulas y "alianzas" que contribuyan a paliarlo tanto como sea posible. 


\section{EL DOLOR ONCOLÓGICO SE DEBE MEDIR}

La intensidad del dolor y el alivio que producen los analgésicos empleados son las variables que más se utilizan para el ajuste de dosis, por lo que llegar a la estandarización en su medición es indispensable para el confort del paciente (46).

Los métodos más útiles usan la información proporcionada por el enfermo como forma de expresión de la intensidad o calidad del dolor. Habitualmente sólo se utiliza la primera de estas medidas en su evaluación, al ser la dimensión más significativa (46), observando lo que ocurre en reposo o cuando se realizan algunos movimientos o maniobras (por ejemplo toser o caminar), sin considerar otras características evaluables, de tipo sensorial (dolor quemante, penetrante o punzante) o afectivo (agotador, atemorizante).

Diversos factores pueden variar el umbral doloroso: la edad (mayor dolor en adultos jóvenes que en ancianos), el sexo (mayor en mujeres), el estado psicológico (mayor en pacientes intranquilos o ansiosos) (47).

Los informes subjetivos de dolor son sin duda los métodos más usados en la evaluación del dolor (48). Se basan en el informe que el paciente realiza, generalmente de la intensidad del dolor, y pueden ser de diferentes tipos:

1. Escala descriptiva simple: son escalas verbales que clasifican al dolor en cuatro, cinco o más categorías, como por ejemplo: Insoportable, Intenso, Moderado, Leve o Ausente, y que muchas veces se confrontan con otras escalas, también descriptivas, del alivio producido por el tratamiento. Este es el método que más se acerca a lo cotidiano, son fáciles de usar y de comprender por los pacientes.

2. Otra variante de escalas categorizan el dolor de acuerdo a la necesidad de analgesia (sin dolor, dolor que no requiere analgesia, y dolor que requiere analgesia), pero no presentan ventajas o limitaciones en relación a la escala verbal simple (49).

3. Escala Visual Analógica (EVA) $(46,49)$ : consiste en una línea recta de $10 \mathrm{~cm}$ de longitud, con las leyendas "Sin dolor" y "Dolor máximo" en cada extremo. El paciente debe anotar en la línea el grado de dolor. La EVA es de uso universal. Es un método relativamente simple, que ocupa poco tiempo, aun cuando requiere de un cierto grado de comprensión y de colaboración por parte del paciente. Tiene buena correlación con las escalas descriptivas, buena sensibilidad y es fácilmente reproducible $(46,50,51)$.

4. Escalas numéricas: se emplean con mucha frecuencia cuando no disponemos de una EVA, en sustitución de aquella o cuando el paciente no es capaz de en- tender o transmitir su dolor con ella. Con estas escalas establecen rangos de 0 a 10 (frecuente en nuestro país), o de 0 a 100 (en países anglosajones).

Se acepta como necesario mantener el dolor habitual o usual en rangos de dolor "moderado-leve", con EVA menor de $4 \mathrm{~cm}$, o escala numérica inferior a 5 ó 50 según la escala utilizada y el rango establecido. Cuando no es posible conocer el dolor "usual", se realizan mediciones a horarios fijos marcando la diferencia entre el dolor inicial y el medido en el momento de la consulta, que indicará con mayor aproximación el grado de analgesia logrado en el tiempo (SPID = score pain intensity difference).

Además de la medición, la observación de la conducta dolorosa es especialmente útil para evaluar el dolor del paciente oncológico, así como para evaluar la respuesta al tratamiento empleado (52). Los índices más utilizados para la evaluación tienen relación con la actividad diaria del paciente, la actividad laboral, el patrón de sueño, la actividad sexual, la alimentación, los signos de dolor (queja, facies), la limitación funcional, las alteraciones en el ánimo y la afectación de las relaciones personales. Si bien estas medidas no cuantifican directamente el dolor, proporcionan datos objetivos que sirven para evaluar la respuesta al tratamiento analgésico o la necesidad de drogas coadyuvantes (sedantes o antidepresivos).

\section{DERIVACIÓN PRECOZ AL ESPECIALISTA DEL DOLOR PREVISIBLEMENTE REFRACTARIO}

Entre el 10 y el 15\% de los pacientes oncológicos van a manifestar una respuesta pobre o inadecuada a los tratamientos farmacológicos habituales (53). Estos pacientes, que no van a obtener un alivio completo o aceptable de su dolor, presentarán de forma mantenida un valor en la Escala Analógica Visual por encima de $7 \mathrm{~cm}$ a pesar de los preceptivos cambios de tratamiento, por lo deberían establecerse signos de alerta que sean capaces de identificarlos (54) y permitan ser derivados precozmente a los centros especializados (55).

La prevalencia del dolor en el paciente oncológico es elevada, dependiendo sobre todo del estadio evolutivo y, aunque en un porcentaje elevado cercano al $90 \%$ es posible conseguir un alivio aceptable siguiendo los protocolos farmacológicos habituales (6), no debemos olvidar que algunos de ellos sufrirán dolor intenso con gran afectación de su calidad de vi- 
da, manteniendo una situación inaceptable que los medios analgésicos comunes no consiguen resolver (7). El dolor oncológico tiene una etiología variada que hay que estudiar exhaustivamente; su origen puede estar ligado al proceso tumoral, al tratamiento oncológico o simplemente a la pérdida de eficacia de los fármacos opiáceos, como señalan algunos autores $(9,10)$. Pero hay multitud de factores que pueden interferir en la eficacia de la terapia analgésica: el estrés emocional, el consumo de sustancias tóxicas, la depresión... Estas causas también deben ser investigadas, reconocidas y evitadas o tratadas de forma coadyuvante (6).

El dolor que no responde a los medios habituales de analgesia se le conoce con el nombre de dolor refractario o dolor de difícil control. Conviene establecer claramente esta definición, identificar a los pacientes con celeridad y buscar rápidamente la alternativa idónea. En enfermos con metástasis óseas destructivas o tumores infiltrantes de estructuras nerviosas, la radioterapia siempre debe considerarse de primera línea y urgente. Algunos autores encuadran erróneamente en este grupo a cuadros que cursan con dolor irruptivo, pero siempre la presencia de este tipo de dolor debe alertarnos sobre la existencia de un cuadro clínico posiblemente complicado de manejar (56). En cualquier caso es esencial hacer una evaluación precoz, para que una vez se haya identificado el problema iniciar la alternativa terapéutica más adecuada (57-64).

Se debería establecer un algoritmo de tratamiento de este síndrome:

1. Utilización de coadyuvantes.

2. Utilización de los fármacos opioides en dosis altas.

3. Rotación de opioides.

4. Medidas específicas paliativas: esteroides, radioterapia, cirugía paliativa, vertebroplastia...

5. Utilización de opioides por vía subcutánea.

6. Utilización de fármacos poco comunes: inhibidores del N-metil-D-aspartato (NMDA)...

7. Estimulación eléctrica transcutánea.

8. Bloqueos nerviosos tanto periféricos como de plexos. Bloqueos simpáticos.

9. Radiofrecuencia de nervios, ganglios dorsales, simpático y centrales.

10. Estimulación eléctrica medular y supramedular.

11. Infusiones intratecales de fármacos tanto opioides como anestésicos locales, clonidina, ketamina, baclofeno, midazolam, betametasona y ziconotida.

12. Cirugía ablativa.

\section{DISPONIBILIDAD UNIVERSAL DE TODOS LOS OPIOIDES}

Existen múltiples formas y etapas para tratar el dolor por cáncer. En primer lugar y como en cualquier otro tipo de dolor, hay que considerar el tratamiento etiológico, es decir, intentar eliminar o paliar la fuente del dolor mediante tratamiento oncológico, ya sea cirugía, radioterapia o quimioterapia. Cuando la causa no puede ser eliminada o paliada con estos métodos, existe un amplio grupo de fármacos para controlar el dolor; en caso de que estos fármacos fracasen hay otros procedimientos terapéuticos que pueden aliviar el paciente y mejorar su calidad de vida. Estas técnicas deben quedar reservadas para enfermos que presentan dolor refractario a las terapias farmacológicas comunes.

Para aplicar adecuadamente los tratamientos analgésicos la Organización Mundial de la Salud (OMS) propuso en el año 1986 una escalera analgésica en la que se recomendaba un tipo u otro de fármaco dependiendo de la intensidad del dolor.

Siguiendo la graduación de intensidad del dolor, los fármacos que se recomiendan son:

- Nivel 1: analgésicos no opioides tales como Aspirina, paracetamol y anti-inflamatorios no esteroideos (AINE).

- Nivel 2: opioides débiles tales como el tramadol, codeína y dextropropoxifeno.

- Nivel 3: opiáceos potentes tales como la morfina y otros opioides (oxicodona, hidromorfona, fentanilo o metadona) que no tienen techo terapéutico y por tanto es posible incrementar sus dosis en función de la intensidad del dolor.

Los opioides son fármacos seguros y fáciles de utilizar. A pesar de la aparición de nuevas galénicas por distintas vías, la vía oral sigue siendo la primera opción terapéutica. Las formulaciones retardadas aportan comodidad de uso y mejoran el cumplimiento terapéutico. Otras vías, como la transmucosa, proporcionan la ventaja de su rápida absorción y consecución de elevados picos plasmáticos, evitando el primer paso hepático. La vía transdérmica aporta la característica contraria y mantiene dosis constantes en sangre evitando los picos plasmáticos. Las presentaciones inyectables para uso intravenoso o intratecal se reservan para los casos complejos en los que han fallado los otros tratamientos.

Es esencial que el paciente con dolor oncológico tenga acceso universal y sin trabas a todas las formulaciones de opioides existentes en el mercado.

Los "teóricos" riesgos sociales de uso indebido de estos productos ha motivado que su empleo se vea so- 
metido a una utilización "vigilada" mediante una dispensación especial que requiere una receta específica y cuantificada. Afortunadamente en los últimos años se han dulcificado mucho las trabas administrativas a la dispensación de opiáceos y se han producido grandes avances en la disponibilidad de estos fármacos. Además han aparecido nuevos productos cada vez más potentes y efectivos y formulaciones novedosas que aportan mejores alternativas para minimizar sus efectos secundarios. Si embargo aún hoy es posible encontrar ciertas limitaciones y barreras que pueden identificarse tanto en los facultativos, las enfermeras y los farmacéuticos como en los propios enfermos y sus familiares. Como consecuencia en muchas ocasiones estos fármacos se infrautilizan o infradosifican con gran perjuicio para el paciente.

A pesar de los tratamientos disponibles, aún existen barreras que hacen que el dolor oncológico a menudo sea infravalorado e infratratado. Existen aún numerosas barreras que hay que superar para tratar el dolor de la forma más adecuada posible. Entre las más importantes se incluyen:

- El conocimiento profesional: mejorar el nivel de conocimientos de los profesionales y dar la debida importancia al tratamiento de todos los síntomas acompañantes del cáncer. Los profesionales deben dar al dolor la importancia debida y ser capaces de reconocer y evaluar su intensidad para aplicar el tratamiento más adecuado. Estos profesionales deben tener acceso a una actualización permanente tanto en los conocimientos farmacológicos como en las habilidades del manejo analgésico.

- Falta de comunicación por parte de los pacientes: se debe evitar el miedo del paciente a comunicar la intensidad del dolor por creer que esto puede desviar la atención del médico y descuidar el tratamiento del tumor. En ocasiones no quiere hacer creer al médico que está peor. La errónea creencia del paciente con cáncer de que la presencia de dolor significa proximidad a la muerte, puede dificultar una comunicación adecuada de la queja dolorosa.

- Miedo a volverse adicto: la mala prensa de los opioides como drogas aditivas hace necesario recalcar que no existe riesgo de adicción cuando se usan correctamente para aliviar el dolor.

- Miedo de los efectos secundarios: muchos pacientes temen los efectos secundarios de los opiáceos que los relacionan con las "drogas" de uso ilegal. Piensan que pueden perder el control, quedarse dormidos, estar "drogados" y no poder comunicarse con sus familiares y amigos o volverse dependientes de la medicación. Incluso algunos enfermos y familiares piensan que pueden acortar la vida y tratan de retrasar su uso.

\section{IMPLICACIÓN DE TODOS LOS PROFESIONALES EN EL TRATAMIENTO DEL DOLOR}

Todos los profesionales sanitarios que están en contacto con el paciente oncológico: médicos de atención primaria, especialistas, enfermeras, psicólogos, trabajadores sociales..., deben sentirse implicados en el tratamiento de su dolor.

El abordaje del paciente con dolor, máxime si este dolor es de origen oncológico, es un tema complejo "el cáncer es una enfermedad que afecta a la persona, a la familia y al entorno social del paciente" (Jovell A, 2004) de ahí la necesidad de un enfoque global al mismo.

El paciente debe ser atendido de forma holística, integral, ya que el dintel de percepción del dolor se liga íntimamente a ello y obliga a contemplar el aspecto subjetivo inherente a este síndrome. Este enfoque requiere la sensibilidad del profesional implicado en el tratamiento: oncólogos, anestesiólogos, paliativistas, médicos de atención primaria..., y en algunos casos psicólogos y equipos de soporte social específico, como promueve una guía elaborada por la Asociación Americana del Dolor (20) y que defiende el enfoque multidisciplinar del tratamiento del dolor como la mejor herramienta dirigida al bienestar del paciente y a su adherencia al tratamiento.

El especialista de Atención Primaria aspira a cuidar al paciente hasta su muerte, siendo importante la continuidad de cuidados y enfocados estos como un todo, le sitúan en una posición privilegiada ante este problema. Estos profesionales, adquieren cada día un mayor adiestramiento en el manejo de estos pacientes gracias a la estrecha relación con los Equipos de Atención Domiciliaria de Cuidados Paliativos, en los que encuentran el apoyo especializado necesario.

La atención multidisciplinaria requiere de un equipo en el que se integran enfermería, el trabajador social, los psico-oncólogos y los farmacéuticos entre otros. El $75-90 \%$ de los pacientes con dolor oncológico están adecuadamente tratados, pero aún persiste un importante número de enfermos infratratados, sobre todo del sexo femenino, con mal estado general o con antecedentes de drogadicción (65).

Con la progresión del cáncer aumenta el disconfort y la incapacidad del paciente, comportando el deterioro del rol familiar, a veces la pérdida del empleo y en síntesis, alterando su propio sentido de identidad, autoestima o autosuficiencia. Finalmente se afecta la esfera espiritual y en definitiva todo su entorno bio-psico-social. La meta del control óptimo del dolor implica prolongar la supervivencia, maximizar el confort y opti- 
Rev. Soc. Esp. del Dolor, Vol. 18, N. ${ }^{2}$ 2, Marzo-Abril 2011

mizar la función, meta no siempre alcanzable. Los aspectos más importantes en su manejo son la evaluación, su tratamiento y el seguimiento posterior. El tratamiento debe ser precoz y no se debe demorar, por lo que el diagnóstico y el tratamiento se deben instaurar simultáneamente (66).

Hoy día el farmacéutico debe integrarse dentro del equipo multidisciplinar tanto en la faceta de experto en la biodisponibilidad e interacciones de los fármacos utilizados en pluritratamiento, como en la faceta de colaborador en la investigación de nuevos esquemas y vías de utilización (67).

El dolor oncológico se asocia siempre a variables psicosociales y dependientes de la personalidad del paciente que hay que tener en cuenta.

El neuroticismo, la introversión-extraversión, el distrés psicológico, la ansiedad, la depresión, el apoyo social, la existencia de estrategias de afrontamiento; por ello el abordaje desde una perspectiva cognitivo-conductual, o modelos psico-educativos y la psicoterapia de apoyo, pueden ser herramientas de gran trascendencia para devolver la calidad de vida al paciente. Encuadrada en el tratamiento multidisciplinar, como una terapia complementaria que facilita y se suma al efecto positivo de otras terapias más centradas en los aspectos físicos de la enfermedad, mejora los componentes de sufrimiento asociado a la experiencia del dolor y que repercuten en la calidad de vida y crea problemas concomitantes como trastornos del sueño, trastornos alimentarios o alteraciones sexuales. Esta herramienta sirve también de ayuda para revertir la depresión que en ocasiones puede acompañarse incluso, con ideación de suicidio (68).

El dolor en cualquiera de sus facetas, agudo o crónico, es inadecuadamente valorado y tratado por múltiples razones (culturales, creencias y actitudes educacionales, políticas, religiosas y de logística). El tratamiento inadecuado tiene repercusiones importantes fisiológicas, sicológicas, sociales y familiares.

En sentido opuesto, el manejo adecuado del dolor se liga al desarrollo sanitario de un país. Pero para llegar a una situación aceptable es necesario derrumbar barreras que aún permanecen arraigadas en nuestra sociedad como son: el miedo a la adicción a opioides, a la posible tolerancia y a los efectos secundarios, relegando su uso para las "etapas finales de la vida". Así se crea una "opio-fobia", basada más bien en una "opioignorancia" que atenta contra el derecho a la salud y el bienestar de los pacientes (69).

Los obstáculos a vencer a nivel internacional implica (69):

1. Educar al personal sanitario que atiende estos pacientes.
2. Crear estándares universales en el tratamiento del dolor por parte de los profesionales.

3. Promover reformas legislativas.

4. Establecer políticas de apoyo del uso y disponibilidad de opioides.

5. Proveer universalmente las distintas presentaciones de opioides.

6. Promover programas de control del dolor en todas las naciones, independientemente de los recursos.

7. Perseverar en el activismo por parte de la OMS, las asociaciones y las sociedades internacionales a favor del control del dolor.

Para terminar, consideramos útil seguir las recomendaciones de la Guía de Práctica Clínica de la NCCN (23), del Instituto del Cáncer Americano, respecto al Código de Educación del Paciente y Familia en el tratamiento del dolor por cáncer, publicadas en 2009 , y referida en la Introducción de este documento.

El derecho del paciente al control permanente del dolor y el adecuado tratamiento de los efectos secundarios que pudieran acontecer, requiere un teléfono de contacto del equipo de profesionales implicados en su cuidado. Se debe finalizar con una recomendación de precaución en el uso de los analgésicos opioides, que deben ser administrados cómo y cuándo se prescriben y solo a la persona a la que se le prescribe y que no debe influir negativamente en el miedo a su uso por falsos mitos. Se debe insistir en la necesidad de expresar verazmente y sin reservas, la existencia e intensidad de dolor a los profesionales que le atienden.

\section{NUEVAS ESTRATEGIAS EN DOLOR. EL PROBLEMA DEL DOLOR IRRUPTIVO}

El dolor irruptivo representa una de las mayores dificultades por las que el dolor oncológico a menudo no llega a tener un control adecuado; este tipo de dolor precisa de un abordaje específico desde el momento de su diagnóstico.

Se define como dolor irruptivo, cualquier exacerbación de dolor que presenta un paciente con un dolor de base que está controlado con tratamiento adecuado. El dolor puede ser espontáneo o estar desencadenado por los movimientos, esfuerzos o ciertas maniobras (defecación, tos,...) siendo catalogado, en este caso, como dolor incidental. Si el dolor aparece sin una causa desencadenante clara, se denomina espontáneo. En los últimos años se ha definido una nueva categoría de dolor irruptivo, que anteriormente estaba incluida en el 
dolor espontáneo. La aparición de dolor coincidiendo con el final del efecto analgésico del fármaco, por este motivo se ha denominado, por fallo al final de dosis (70).

Según las series consultadas la prevalencia de dolor irruptivo oscila entre el 52 y el $89 \%$ de los pacientes con dolor; esta gran variabilidad es debida a la falta de consenso en la definición, aunque también influye el ámbito donde se haya realizado el estudio (hospitalización versus atención primaria), e incluso del país donde se haga la encuesta (71).

Se considera dolor irruptivo a una exacerbación de dolor, de instauración rápida, menos de 3 minutos, de intensidad elevada y una duración entre 15-30 minutos. Es raro que un dolor irruptivo tenga una duración superior a 60 minutos. Según la fisiopatología, se identifica como somático (33-45\%), visceral (20-29\%), neuropático (9-33\%) y mixto (16-20\%). El dolor irruptivo está producido por el cáncer en el $75 \%$ de los casos aproximadamente, un $20 \%$ son secundarios a la terapia antineoplásica y entre un $4-19 \%$ no hay una correlación con la enfermedad ni el tratamiento. La tercera parte del dolor irruptivo puede ser secundario al efecto de final de dosis (72).

Realizar un diagnóstico correcto es la clave para poder realizar un tratamiento correcto. La clave para el diagnóstico es identificar la causa, que va a permitir catalogar el dolor irruptivo como incidental o por fallo de dosis.

La dificultad de manejo del dolor irruptivo es de tal magnitud, que obliga a realizar las pruebas complementarias necesarias para poder realizar un correcto diagnóstico diferencial.

La estrategia terapéutica es completamente diferente si la causa es una metástasis ósea, una distensión intestinal o uretral o por final de dosis. Por tanto, la evaluación del dolor irruptivo debe contemplar los mismos principios que la evaluación de cualquier dolor, y para ello es necesario identificar todos los aspectos físicos, emocionales, sociales y valores asociados (73).

Según la clasificación pronóstica del dolor (Edmonton Staging System) (74), el dolor irruptivo es considerado de mal pronóstico o de tipo II. Además de utilizar las escalas de medición de la intensidad del dolor, como la Escala Visual Analógica (EVA) u otras, es fundamental analizar los factores desencadenantes del dolor, tiempo de instauración y duración, características del dolor, factores que promueven alivio del mismo y la correlación entre el dolor y la dosificación de la pauta analgésica.

El objetivo de la estrategia terapéutica es doble, tratar y prevenir. Realizar un tratamiento que disminu- ya el dolor instaurado de forma rápida, y aún más importante, incidir directamente sobre los factores desencadenantes del dolor realizando una acción preventiva.

La estrategia terapéutica debe incluir:

- Medidas dirigidas a tratar la causa del dolor.

- Optimizar el tratamiento farmacológico.

- Medidas intervencionistas.

- Otras medidas.

- Medidas dirigidas a tratar la causa del dolor. Una vez identificada la causa, es necesario evaluar si existe un tratamiento que sea capaz de modificarla. Si es el propio tumor la causa directa o indirecta del dolor, por infiltración o compresión de una estructura, es lógico pensar en la utilización de citostáticos, siempre y cuando actúen sobre tumores sensibles. La radioterapia estaría indicada en lesiones óseas producidas por metástasis con o sin fractura asociada y en situaciones que precisen descompresión neurológica urgente (compresión medular,...). La cirugía podría ser útil en situaciones álgicas secundarias a fracturas o con efecto compresivo.

- Optimizar el tratamiento farmacológico. La optimización del tratamiento farmacológico es la medida más accesible y la más utilizada, pero a menudo ofrece resultados poco satisfactorios bien por la aparición de la respuesta analgésica pasada la crisis de dolor, bien por la aparición de efectos indeseables ligados a la acción "pico" del fármaco empleado, como la somnolencia, bien a la no prescripción de pautas de rescate con fármacos y dosis adecuadas.

Por estos motivos, al diseñar una pauta de tratamiento para un dolor irruptivo se deben tener en cuenta las siguientes premisas: utilizar un fármaco con un tiempo de vida media corto y, escoger una vía de administración adecuada con la finalidad de poder evaluar la respuesta analgésica en poco tiempo (75). Los opioides como la morfina y el fentanilo han demostrado según el tipo de presentación, que son efectivos para este tipo de dolor (75).

La administración de estos opioides por vía transmucosa, sublingual e intranasal han demostrado efectividad similar a la vía parenteral (endovenosa, subcutánea) o espinal (76-78). Es importante remarcar que cualquier protocolo analgésico para el dolor oncológico no es completo si no dispone de una pauta para el dolor basal y otra para las crisis de dolor, las denominadas dosis de rescate. En el dolor irruptivo este hecho es fundamental.

Para prevenir el dolor irruptivo, sea espontáneo o debido a un fallo por final de dosis, existen diferentes estrategias: aumentar la dosis total diaria, establecer pautas de rescate en horarios fijos, realizar una rota- 
ción de opioide si aparecen efectos secundarios al subir dosis para conseguir la analgesia adecuada.

- Medidas intervencionistas. Se definen como medidas intervencionistas aquellas técnicas analgésicas específicas que precisan de un especialista para su realización. Son las realizadas en las Unidades de Tratamiento del Dolor. Los bloqueos de nervio periférico o de plexos, las infiltraciones epidurales, radiculares o musculares, la neuromodulación, el uso de la vía espinal $(79,80)$ y otras ya expuestas en otro apartado de este documento.

-Otras medidas. La fisioterapia para mejorar la función y la afectación muscular o articular, las medidas ortopédicas de apoyo mediante ortesis para modular movimientos o inmovilizar zonas dolorosas, son técnicas efectivas pero a menudo no utilizadas.

La atención psicológica puede ser de utilidad para disminuir el impacto emocional que puede desencadenar el dolor irruptivo, su utilidad también ha sido demostrada en técnicas dirigidas a producir un incremento del umbral del dolor.

\section{ÉTICA. NO SE DEBERÍA ACEPTAR UN DOLOR INTENSO MANTENIDO}

El sufrimiento intenso y continuado de un paciente por dolor oncológico pudiera resultar no ético en nuestro contexto sanitario actual. Nos consta que, en la actualidad y en las diversas comunidades autónomas de nuestro estado, existen profesionales con experiencia y conocimientos suficientes para poder manejar todas las situaciones de dolor difícil.

Se debería garantizar el acceso a las unidades especializadas en tratamiento del dolor a todos aquellos pacientes que padezcan dolor intenso por cáncer que no remita a pesar del manejo inicial.

En concreto proponemos que un dolor oncológico de intensidad de 7/10 o mayor en la Escala Verbal Numérica, o intenso en la Escala Verbal Categórica, se considere un signo clínico de alarma, para que la respuesta sanitaria ante el mismo sea lo más urgente posible.

Más de 30 millones de personas sufren innecesariamente cada año, en todo el mundo, por dolor intenso y otros síntomas. Esta situación no es ética, ya que existen métodos simples y efectivos para paliar el dolor y otros síntomas, que pueden ser aplicados a nivel comunitario con una cobertura de la población adecuada (81).

La magnitud del dolor es tan sólo un factor en el sufrimiento que produce. Se puede tolerar incluso un do- lor intenso si se sabe lo que es, que no implica peligro y que tendrá un final. Un dolor de menor intensidad puede ser mal tolerado y conducir a sufrimiento, si se presume una etiología funesta o un curso indefinido (82). Ya se ha mencionado previamente la relación entre la intensidad del dolor por cáncer y la aparición en el paciente del deseo de que se anticipe su muerte. Por tanto, un manejo adecuado y agresivo del dolor oncológico es la respuesta ética lógica y razonable (83).

El dolor por cáncer se suele evaluar por diferentes motivos: diagnóstico diferencial, factores contribuyentes, evolución etc. Uno de los motivos fundamentales es determinar la intensidad del mismo y su interferencia en las actividades cotidianas (84). Conforme progresa la enfermedad cancerosa, el dolor suele incrementarse en frecuencia de episodios e intensidad $(85,86)$. El consenso internacional establece una cifra de entre el 10 y el $20 \%$ de los pacientes con dolor oncológico que van a presentar cuadros de dolor intenso de difícil manejo y sufrimiento inútil en manos no especializadas $(87,88)$.

La investigación biomédica en el campo del dolor de origen canceroso ha demostrado, hace tiempo, el elevado impacto en las actividades cotidianas que produce un dolor intenso en la Escala Verbal Categórica (nada - leve - moderado - intenso - insoportable), o superior a 7/10 en la Escala Verbal Numérica ( 0 = na$\mathrm{da}, 10=$ el peor dolor imaginable). Es muy probable que un paciente en esta situación no pueda moverse sin acrecentar aún más el dolor. Además probablemente, no podrá conciliar el sueño, y si al final consigue dormir será con un sueño fragmentado y poco reparador (89).

Consideramos, por tanto, que es ético y razonable en nuestro actual contexto sanitario, proponernos como objetivo que todo paciente afecto de dolor oncológico intenso (mayor de 7/10) y continuado, reciba una atención urgente y adecuada. La derivación de estos enfermos a profesionales con experiencia y conocimientos suficientes para manejar estos casos, no debería suponer en principio un reto inabordable para nuestro sistema sanitario.

Consideramos que como profesionales sanitarios tenemos un deber ético de ser competentes en el alivio del dolor por cáncer y de proporcionar una analgesia adecuada. También estamos obligados a no imponer este tratamiento sin consentimiento del paciente.

Los pacientes tienen derecho a esperar de nosotros que haremos todo lo que resulte clínicamente apropiado y necesario para aliviar su dolor. Los profesionales tenemos la obligación moral de alcanzar los conocimientos, habilidades y coraje suficientes para alcanzar este objetivo (90). 


\section{ACCESIBILIDAD A LOS ESPECIALISTAS EN TRATAMIENTO DEL DOLOR}

La mayoría de los pacientes con dolor por cáncer se controlan adecuadamente con la administración de opioides por vía oral o parenteral (10). Algunos de ellos precisan la aplicación de fármacos coadyuvantes, psicofármacos o ayuda psicológica especializada y todos ellos pueden y deben estar bajo el cuidado de su médico de familia o el especialista que sigue la evolución y el cuidado de su enfermedad. Pero aún con todo, un grupo de pacientes (5-10\%) son portadores de cuadros clínicos que hacen suponer que en algún momento van a padecer dolor severo $(91,92)$, en ocasiones insoportable, refractario a toda terapia convencional y que van a necesitar la ayuda de técnicas analgésicas sofisticadas o bien terapia intratecal continua, para lo que es necesario que sean derivados a Unidades Especializadas. Además, un grupo nada desdeñable de pacientes, puede ahorrar gran consumo de analgésicos y sufrimiento $y$ van a mejorar su calidad de vida si se someten prematuramente a alguna de estas técnicas, siempre que esté bien indicada y el momento y el estado del paciente lo aconsejan.

En 1994 se consideraba que aproximadamente la mitad de los pacientes (40-50\%) que padecían dolor por cáncer, no encontraban suficiente alivio a pesar de las terapias analgésicas empleadas (1). Un estudio multicéntrico italiano reciente (92) establece que, aun a pesar de los avances que se han producido en los últimos años, el 25,3\% los pacientes con dolor por cáncer son tratados de forma inadecuada. En este estudio se encontró que existe una amplia variación entre los centros encuestados (que oscila entre el 9,8 y el $55,3 \%$ ); esta diferencia dependía del grado de desarrollo de tratamiento del dolor del centro que recogía el dato y de su posibilidad de utilizar técnicas coadyuvantes. Así se constató que el centro que disponía de acceso rutinario a una Unidad Multidisciplinar del Dolor tenía cifras más bajas.

A pesar de la importante mejora del manejo del dolor del paciente con cáncer a partir de la implantación de la guía clínica para su tratamiento publicado en 1986 por la Organización Mundial de la Salud (OMS) y revisado recientemente (93-95), la falta de alivio en muchos de estos pacientes sigue siendo un problema importante (5).

Como respuesta a este problema, es incuestionable la utilidad de terapias analgésicas especiales, como los bloqueos neurolíticos o la terapia intratecal continua, que requieren Equipos Multidisciplinares entrenados en estas técnicas $(96,97)$.
Todos los estudios epidemiológicos reconocen que un $5-10 \%$ de los pacientes que sufren dolor por cáncer pueden necesitar la ayuda de estas Unidades en algún momento de su evolución $(91,92)$.

Estados Unidos publicó en 1999 una nueva iniciativa de crear un estándar que proporcionara un mecanismo de evaluar la calidad en la práctica y documentación de los centros en la atención al dolor, a través de una Comisión Conjunta de Acreditación de las Organizaciones de la Salud (JCAHO). Esta iniciativa fue evaluada hace cinco años (98), demostrando ser una buena herramienta para adecuar el adecuado manejo del dolor que debía apoyarse en cuatro actuaciones:

1. Declarar el cuidado del dolor como un derecho del paciente.

2. Considerar el problema de la educación y formación específica en el cuidado del dolor.

3. Considerar la necesidad de evaluar el dolor sistemáticamente.

4. Utilizar siempre el tratamiento analgésico más seguro.

Es necesario conocer que cuando un tratamiento analgésico es o se vuelve ineficaz, se convierte en un arma terapéutica insegura, tanto más cuanto menos eficaz se muestre. La primera causa de este principio es la ausencia de techo terapéutico de los opioides. Ante casos de no alivio del dolor, muchos profesionales suben de forma progresiva y en ocasiones injustificada las dosis del fármaco, sin plantearse buscar el motivo que está provocando la falta de respuesta al opioide o bien tantear otras alternativas terapéuticas. Con esta forma de actuar, los efectos secundarios de estos fármacos no tardan en hacerse protagonistas de la situación y el caos terapéutico desborda al enfermo, la familia y al propio profesional.

Las técnicas analgésicas aplicadas en las Unidades del Dolor que han sido descritas en párrafos anteriores, mejoran su efectividad y el beneficio a medio plazo si se emplean precozmente. La ética y el comportamiento racional de los médicos que cuidamos a los pacientes oncológicos deberían indicarnos el preciso momento en el que esta intervención es necesaria. Alargar un tratamiento ineficaz o retrasar una técnica analgésica puede entorpecer e incluso impedir su realización posterior.

En el momento que el médico haya tomado la decisión de derivar un enfermo, la administración debería garantizar que todos ellos, sin distinción de ciudad, región o posibilidades económicas, tuvieran la posibilidad de ser atendidos sin demora en una Unidad Especializada. Actualmente la importante carencia en el tratamiento especializado del dolor que existe en nuestro 
país y la dismetría entre las distintas comarcas exigen un planteamiento global e institucional para mejorar nuestra eficiencia en el tratamiento del dolor refractario.

\section{MIEDO A LOS OPIOIDES: TOLERANCIA, ADICCIÓN, EFECTOS SECUNDARIOS}

La tolerancia es la necesidad de aumentar progresivamente la dosis de una droga para mantener el mismo efecto farmacológico tras administraciones repetidas (99).

La tolerancia se considera un fenómeno farmacodinámico. Se asocia a cambios del comportamiento neuronal frente a estímulos externos por cambios estructurales denominados "plasticidad neuronal". La lesión nerviosa, la inflamación de una zona, la administración de opioides, pueden inducir estos cambios (100).

Nuestro organismo dispone de opioides endógenos. La consecuencia de administrar opioides exógenos, necesarios para controlar un dolor, provoca cambios en los receptores opioides que se caracterizan por una disminución del número de receptores y una estimulación de sistemas pronociceptivos paralelos: NMDA,... (101). Hay estudios publicados que demuestran que los cambios neuronales pueden revertirse con la reexposición de opioides y que en función del tiempo de exposición, debe incrementarse la dosis de algunos opioides (102).

El fenómeno de tolerancia en la práctica clínica, implica la necesidad de aumentar progresivamente las dosis del opioide para mantener el mismo efecto analgésico. Es un proceso intrínseco de los opioides utilizados en la práctica clínica, pero existen diferencias entre las patologías y distintos opioides en relación al tiempo y el grado de desarrollo.

La adicción es un fenómeno psicopatológico dirigido a tomar de forma compulsiva un fármaco para obtener efectos psicológicos (103). Los efectos analgésicos de los opioides se realizan a través del sistema nervioso central, actuando en diferentes áreas que permiten disminuir la percepción de dolor. Como efectos colaterales pueden producir alteraciones neurológicas en forma de trastornos cognitivos, alucinaciones, etc.

El paciente precisa la administración de un opioide única y exclusivamente para controlar su dolor; si obtiene el efecto deseado, disminución o desaparición del dolor, el paciente deseará seguir consumiendo el opioide para seguir percibiendo este beneficio. Este deseo se denomina "dependencia psicológica".
La dependencia psicológica provoca miedo a no disponer del fármaco en el momento que lo precisa y por ello hacen acopio del fármaco antes de hacer un viaje y preguntan con ansiedad si van a tener disponibilidad de él. En realidad el enfermo presenta "miedo anticipatorio" a la reaparición del dolor por falta del fármaco (104). Por el contrario, la adicción es la necesidad de tomar un fármaco de forma compulsiva para obtener un efecto psicológico.

Las series publicadas en referencia a la adicción son limitadas, y en todas se hace referencia a ella como un hecho anecdótico y puntual (103-105). La experiencia de los profesionales permite identificar directa o indirectamente el comportamiento adictivo de un paciente. Directamente por la visita médica: analizando su personalidad, comportamiento, antecedentes por abuso de drogas, etc., e indirectamente mediante el conteo del número de envases prescritos mediante receta.

La existencia de dependencia psicológica por toma de opioides, es un indicador de dolor bien controlado. La evolución de la dependencia puede variar en función de la evolución de la enfermedad. Si mediante los tratamientos específicos se consigue una remisión o curación de la enfermedad, es probable que el dolor disminuya y la necesidad de opioides también. En situaciones de enfermedad avanzada la mayoría de pacientes necesitaran opioides de por vida con una dependencia establecida.

Otro de los miedos en el uso de opioides son los efectos secundarios. Un efecto secundario es la consecuencia indirecta y generalmente adversa del uso de un medicamento o terapia. Los producidos por los opioides, no sólo son una consecuencia de la interacción de estos con los receptores específicos, sino que otros factores pueden influir en su aparición, como la edad, la extensión de la enfermedad, la disfunción de órgano, la interacción con otros fármacos, el tipo de opioide administrado y la vía de administración (106). La gran variabilidad interpersonal en la aparición de estos efectos puede explicarse por las variaciones genéticas de cada individuo.

En la mayoría de casos, los efectos secundarios se pueden prevenir y/o disminuir su intensidad teniendo en cuenta una serie de recomendaciones:

La prescripción de un opioide obliga a (107):

1. Realizar un análisis funcional del paciente: edad, estadio y localización de la enfermedad, hidratación, función renal y hepática, función cognitiva, frecuencia respiratoria, hábito intestinal.

2. Seleccionar el opioide y la dosis adecuados para la intensidad y el tipo de dolor que presenta. 
3. Elegir la vía de administración más adecuada. Según el tipo de opioide la incidencia de algunos efectos indeseables disminuyen, como el estreñimiento.

4. Ajustar las dosis según las necesidades del paciente para conseguir una razón efectividad-seguridad adecuada.

5. Revisar el resto de tratamiento prescrito para poder disminuir al máximo las interacciones farmacológicas. La mayoría de efectos secundarios a nivel del sistema nervioso central, tienen su origen en las interacciones de los opioides con benzodiazepinas, antidepresivos, anticonvulsivantes, etc.

Es necesario realizar un tratamiento individualizado para poder conseguir una buena adherencia terapéutica. El fracaso de una pauta analgésica debido a la aparición de efectos secundarios es una fuente de desconfianza y frustración para un paciente con dolor oncológico.

Los efectos secundarios inducidos por los opioides, pueden clasificarse en 4 grupos (108):

1. Efectos gastrointestinales: náusea, vómito y estreñimiento.

2. Efectos autonómicos: sequedad de boca, retención de orina e hipotensión postural.

3. Efectos sobre el sistema nervioso central (109): somnolencia, fallo cognitivo, alucinaciones, delirium, depresión respiratoria, mioclonías e hiperalgesia.

4. Efectos cutáneos: prurito y sudoración.

La incidencia de los mismos puede variar por varios factores: si el paciente recibe por primera vez tratamiento opioide, o el paciente recibe tratamiento crónico con opioide.

En el paciente que recibe por primera vez tratamiento analgésico con opioides, inicialmente y de forma transitoria, los efectos secundarios gastrointestinales serán los más frecuentes, excepto la constipación que puede mantenerse a lo largo del tratamiento (108). La aparición en esta situación de efectos intensos del sistema nervioso central, obliga ha realizar con urgencia una revisión del caso (dosificación, interacciones farmacológicas, alteraciones metabólicas,...) (109).

Es fundamental realizar un diagnóstico diferencial del efecto secundario que aparece en el curso del tratamiento analgésico con opioides. Es fácil cometer el error de culpar a los opioides de todos los efectos secundarios, aunque se estima que el $50 \%$ pueden tener otra causa

Para disminuir el impacto de los efectos secundarios se deben abordar una serie de medidas (110):

1. Reducción de dosis: es una medida sin riesgo y a menudo necesaria. Estaría indicada si el dolor está bien controlado; implica realizar un seguimiento cercano del paciente y administrar dosis de rescate ajustadas según la necesidad. Puede ser útil en pacientes de edad avanzada (111). Si por el contrario, el dolor no está bien controlado, la reducción de dosis debe completarse con la asociación de otro fármaco o técnica analgésica adyuvante (radioterapia, anestésica, quimioterapia).

2. Tratamiento sintomático: el uso de tratamientos sintomáticos para reducir el efecto secundario, es un tema controvertido. Hay consenso en administrar tratamiento sintomático preventivo al inicio de la terapia con opioides (síntomas gastrointestinales) y para paliar de forma puntual algunos síntomas neuropsicológicos, pero la clave del tratamiento es actuar directamente sobre la causa que lo genera. El uso de tratamientos sintomáticos provoca un mayor número de medicación para el paciente, un mayor riesgo de presentar interacciones farmacológicas y un beneficio escaso a corto plazo.

3. Rotación de opioides: se entiende por rotación de opioides, la substitución del opioide previo por otro distinto con el objetivo de obtener una respuesta más favorable. Este tema también genera controversias, pero se dispone de evidencia científica que esta estrategia es efectiva para algunos efectos secundarios del sistema nervioso central (fallo cognitivo, alucinaciones, mioclonías) y del sistema gastrointestinal (náuseas, vómitos). La base fisiopatológica reside en el grado de estimulación que cada opioide realiza a nivel de los receptores. Se recomienda que la realicen profesionales especialistas o con un alto conocimiento del manejo de fármacos opioides.

4. Vía de administración del opioide: que puede contribuir a una disminución de algunos efectos secundarios como náuseas, vómitos y constipación. En general, la eficacia de la mayoría de opioides es independiente de la vía de administración.

Estas medidas deben de ser complementarias a otras y dirigidas a estabilizar funciones descompensadas del organismo. Por ejemplo, en caso de fallo renal, medidas específicas para mantener la función renal y el equilibrio hidroelectrolítico, en caso de deshidratación, hidratar, medidas para corregir la hipoxemia, etc. La combinación de unas y otras deben de ser suficientes para poder corregir la mayoría de los efectos secundarios. El recurso de utilizar opioides antagonistas como la naloxona, no debe descartarse. Su indicación en situaciones de urgencia es correcta, mediante una protocolización de uso. 


\section{RADIOTERAPIA PALIATIVA EN FRACCIÓN ÚNICA. EVIDENCIAS DE EFICACIA}

La radioterapia en fracción única cumple los siguientes objetivos y principios: implicar al paciente en la toma de decisiones de forma rápida y un ahorro en el tiempo de espera que es fundamental para:

- Evitar retrasos en el inicio del tratamiento radioterápico.

- Tratamientos de duración corta (esquemas de eficacia comprobada).

En algunos casos, la paliación no debe excluir necesariamente la curación. La irradiación paliativa no debería consumir más recursos que los necesarios para alcanzar su objetivo.

Su indicación principal es el dolor grave por metástasis óseas. Las metástasis óseas constituyen la causa más frecuente de dolor crónico severo. Las localizaciones más frecuentes son: la columna lumbar, la columna dorsal, la pelvis, las costillas, los fémures y la calota.

Los tumores que más metastatizan en el hueso son, por orden de frecuencia: mama, próstata, tiroides, riñón y pulmón.

La RT paliativa consigue un alivio sintomático en el 60-90\% de los pacientes.

Indicaciones en general: a) alivio del dolor; b) metástasis óseas; c) adenopatías dolorosas; d) infiltración de tejidos blandos; e) dolor neuropático; y f) dolor por síndrome obstructivo.

También se utiliza para prevenir las complicaciones y eventos óseos inesperados como fracturas patológicas o síndrome de compresión medular.

La radioterapia es el tratamiento de elección en la metástasis ósea dolorosa porque produce:

- Alivio rápido del dolor (70-80\%).

- Pico de alivio a las 4 semanas.

- Mediana de duración 3 meses.

Esquemas de tratamiento:

-8 Gy en una sola fracción.

-20 Gy en 5 fracciones.

-30 Gy en 10 ó 15 fracciones.

En las metástasis óseas no complicadas por fractura patológica o compresión medular, en las que la intención de tratamiento sea exclusivamente antiálgico, se recomienda una fracción única de 8 Gy.

Este tratamiento tiene demostrada su efectividad con un elevado nivel de evidencia (112-114).

- Nivel I de evidencia.

- Grado de recomendación A.

No disponemos de suficientes evidencias para efectuar recomendaciones sobre esquemas fraccionados (20/5 fracc.; 30/10 fracc.) para objetivos paliati- vos que no sean solo el analgésico (115), sus indicaciones son:

- Pacientes con metástasis únicas y larga expectativa de vida.

- Prevención y tratamiento de la compresión medular.

- Prevención y tratamiento de fracturas patológicas (116).

- Tratamiento de las masas de partes blandas asociadas a lesiones óseas.

La radioterapia es efectiva para la paliación, aportando un $80-90 \%$ de mejorías en un 50\% de los casos puede haber recaída, aunque en este caso el paciente puede ser nuevamente evaluado para realizar otro tratamiento. El esquema de tratamiento óptimo no pudo ser determinado (115).

Se pueden emplear dosis mayores en situaciones especiales como metástasis solitarias o con largo intervalo libre de enfermedad: dosis: $2.000 \mathrm{cGy} / 5$ fracciones, $3.000 \mathrm{cGy} / 10$ fracciones, $3.500 \mathrm{cGy} / 14$ fracciones.

En un estudio de metaanálisis reciente (117) se evaluaron 3.260 pacientes seleccionados de 7 estudios con dosis única de 8 Gy (8-10 Gy) en comparación con dosis fraccionada (mediana 20 Gy en 5 sesiones, esquemas 20 y 30 Gy en 5-10 s.). Los resultados en términos de RC son similares: 32,4 vs. 32,9\%. La respuesta global mejor fue para dosis única: $62,1 \mathrm{vs} .56,7$ $(\mathrm{p}=0,04)$.

En el subanálisis restringido a los pacientes evaluados no hubo diferencias significativas. Hubo más frecuencias de re-irradiaciones en el grupo de dosis única.

En conclusión la RT de las metástasis óseas no complicadas debe realizarse mediante dosis única de $8 \mathrm{~Gy}$ (118).

\section{QUIMIOTERAPIA PALIATIVA}

La quimioterapia constituye una herramienta terapéutica única e importante dentro del contexto del tratamiento multidisciplinario del cáncer. Al igual que la radioterapia, la cirugía y los tratamientos con fármacos analgésicos opiáceos y no opiáceos, la quimioterapia puede tener un efecto paliativo importante, ya que si no consigue una erradicación de la enfermedad al menos puede aportar un alivio significativo de los síntomas.

El dolor producido por una neoplasia puede ser debido al efecto directo de la enfermedad por compresión o infiltración de los tejidos y sus terminaciones nerviosas. Este componente meramente físico, por la expansión y crecimiento tumoral, coexiste con el dolor causado por la reacción inflamatoria que induce el propio 
tumor y que causa sensibilización de las terminaciones nerviosas anejas.

La quimioterapia como tratamiento citotóxico/citorreductor puede aliviar el dolor de forma importante, en particular el dolor óseo en pacientes con cáncer de mama o mieloma múltiple. Es menos efectiva cuando el tumor primario es de próstata, riñón o de pulmón (no microcítico) (119). Un caso paradigmático en oncología ha sido la incorporación del agente quimioterápico gemcitabina para el cáncer de páncreas. Este tipo de cáncer afecta a importantes plexos nerviosos debido a la profusa inervación tisular del órgano que hace que sea el prototipo de los cánceres con intenso dolor tumoral. La aparición de este fármaco en nuestros hospitales ha hecho que el curso clínico de los pacientes con este tumor cambiara fundamentalmente no en supervivencia, que la hay con supervivencias de unas semanas, sino en intensidad del dolor y en consumo de analgésicos. En definitiva este fármaco ha aportado al enfermo de cáncer de páncreas un aumento notable en su calidad de vida. Recientemente, en un estudio clínico en pacientes con cáncer de páncreas se añadió a la gemcitabina la capecitabina, otro fármaco quimioterápico y se demostró que los dos juntos beneficiaban más que la gemcitabina sola en cuanto al dolor, la intensidad de este y el consumo de analgésicos (120). El ejemplo del cáncer de páncreas es una muestra de la contribución de la quimioterapia al alivio del dolor en la mayoría de cánceres y a la mejora de la calidad de vida de nuestros pacientes conjuntamente con agentes terapéuticos de otras especialidades en un contexto de tratamiento multidisciplinario.

La capacidad de la quimioterapia en reducir el volumen tumoral por su propiedad citotóxica, ya sea en un periodo de días o de semanas, es una herramienta muy valiosa para combatir el dolor canceroso conjuntamente con todas las herramientas con que cuenta hoy en día el médico.

Aunque no se puedan considerar propiamente agentes quimioterápicos, los bisfosfonatos proporcionan al médico oncólogo una herramienta antihiperalgésica excelente al haber demostrado que cuando se administran conjuntamente con la quimioterapia aportan una marcada reducción en el dolor óseo inducido por las metástasis así como en la reducción de fracturas patológicas por erosión del hueso por el propio cáncer (121). Las metástasis óseas afectan a un $65-75 \%$ de los pacientes con cáncer de mama o de próstata, de ahí la importancia de poder administrar estos fármacos en enfermos con estos tipos de cáncer. En el tratamiento del cáncer uno debe de seguir la norma del dicho popular que dice "curar a veces, aliviar a menudo, confortar siempre".
CORRESPONDENCIA:

Luis Miguel Torres Morera

Servicio de Anestesiología y Reanimación

Hospital Universitario Puerta del Mar

Avda. Ana de Viya, 21

11009 Cádiz

e-mail: 1mtorres@arrakis.es

\section{BIBLIOGRAFÍA}

1. Cherny NI, Portenoy RK. The management of cancer pain. CA Cancer J Clin1994;44:263-303.

2. Zhukovsky IDS, Gorowski E, Hausdorff J, Napolitano B, Lesser M. Unmet analgesic needs in cancer patients. J Pain Symptom Manage 1995;10:113-9.

3. Zech DF, Grond S, Lynch J, Hertel D, Lehmann KS. Validation of World Health Organization guidelines for cancer pain relief. Pain 1995;63:65-76.

4. Serlin RC, Mendoza TR, Nakamura Y, Edwards KR, Cleeland CS. When is cancer pain mild, moderate or severe? Grading pain severity by its interference with function. Pain 1995;61:277-84.

5. Levy M. Pharmacologic treatment of cancer pain. $\mathrm{N}$ Engl J Med 1996;335:1124-32.

6. Portenoy RK, Lesage P. Management of cancer pain. Lancet 1999;353:1695-700.

7. Whitecar PS, Jonas AP, Clasen ME. Managing pain in the dying patient. Am Fam Physician 2000;61:755-64.

8. Sepulveda C, Marlin A, Yoshida T, Ulrich A. Palliative care: the World Health Organization's global perspective. J Pain Symptom Manage 2002;24:91-6.

9. Foley KM. Pain assessment and cancer pain syndromes. In: Doyle D, Hanks GWC, Calman K, Cherny $\mathrm{N}$, editors. Oxford textbook of palliative medicine. 3rd ed. New York: Oxford University Press; 2003.

10. Bruera E, Kim HN. Cancer pain. JAMA 2003;290:2476-9.

11. Khosravi Shahi P, Del Castillo Rueda A, Pérez Manga G. Manejo del dolor oncológico. An Med Interna 2007;24:554-7.

12. Burton AW, Fanciullo GJ, Beasley RD, Fisch MJ. Chronic pain in the cancer survivor: a new frontier. Pain Med 2007;8:189-98.

13. Wandler E, Kramer EL, Sherman O, Babb J, Scarola J, Rafii M. Diffuse FDG shoulder uptake on PET is associated with clinical findings of osteoarthritis. AJR Am J Roentgenol 2005;185:797-803.

14. Sharpe M, Strong V, Allen K, Rush R, Postma K, Tulloh A, et al. Major depression in outpatients atten- 
ding a regional cancer centre: screening and unmet treatment needs. Br J Cancer 2004;90:314-20.

15. Reich M. Depression and cancer: recent data on clinical issues, research challenges and treatment approaches. Curr Opin Oncol 2008;20:353-9.

16. Chen ML, Chang HK. Physical symptom profiles of depressed and nondepressed patients with cancer. Palliat Med 2004;18:712-8.

17. Novy D, Berry MP, Palmer JL, Mensing C, Willey J, Bruera E. Somatic symptoms in patients with chronic non-cancer-related and cancer-related pain. J Pain Symptom Manage 2005;29:603-12.

18. Strong V, Waters R, Hibberd C, Murray G, Walker J, McHugh G, et al. Depression care for people with cancer; a randomized trial of a complex intervention. Lancet 2008;5:8-10.

19. Walker J, Cassidy J, Sharpe M. The second Symptom Management Research Trial in Oncology (SMaRT Oncology-2): a randomised trial to determine the effectiveness and cost-effectiveness of adding a complex intervention for major depressive disorder to usual care for cancer patients. Trials 2009; 10:18.

20. Gordon DB, Dahl JJ, Micsskanski C, McCarberg B, Todd KH, Paice JA, et al. American Pain Society recommendations for improving the quality of acute and cancer pain management. American Pain Society Quality of Care Task Force. Arch Intern Med 2005;165:1574-80.

21. Cleeland CS, Janjan NA, Scott CB, Seiferheld WF, Curran WJ. Cancer pain management by radiotherapists: a survey of radiation therapy oncology group physicians. Int J Radiat Oncolo Biol Phys 2000; 47:203-8.

22. Steenland E, Leer JW, van Houwelingen H, Post WJ, van den Hout WB, Kievit J, et al. The effect of a single fraction compared to multiple fractions on painful bone metastases: a global analysis of the Dutch Bone Metastasis Study. Radiother Oncol 1999;52: 101-9.

23. NCCN Clinical Practice Guidelines in OncologyTM (v1) 2009. Adult cancer pain

24. Deng G, Cawhisth BR. Integrative oncology; complementary therapies for pain anxiety and mood disturbance. C A Cancer. J Clin 2005;55:109-16.

25. Zech DF, Griund S, Lynch J, Hertel D, Lehmann KA. Validation of World Health Organization Guidelines for Cancer Pain relief; a 10 year prospective study. Pain 2005;63:65-76.

26. Casinello J, Camps C. Guía rápida de tratamiento médico del dolor oncológico. Madrid: SEOM; 2005.

27. Fuentes, Canales, Vayrada. Tratamiento de soporte en oncología radioterápica. Madrid: SEOR; 2003.

28. Ministerio de Sanidad y Consumo, SECPAL. Guía de criterios de calidad en cuidados paliativos. Madrid: Ministerio de Sanidad y Consumo; 2002.

29. Abordaje integral del paciente oncológico avanzado en atención primaria. Atención Primaria. semFYC 2006;38:1-23.

30. Recomendaciones Rec (2003) 24 del comité de Ministros de los estados miembros sobre organización de cuidados paliativos. Madrid: Agencia de Calidad del sistema Nacional de Salud. Ministerio de Sanidad y Consumo; 2005.

31. Estrategia Nacional de Salud en Cuidados Paliativos. Madrid: Ministerio de Sanidad y Consumo; 2007. p. 35.

32. Roth AJ, Massie MJ. Anxiety and its management in advanced cancer. Curr Opin Support Palliat Care 2007;1:50-6.

33. Breitbart W. Psychiatric management of cancer pain. Cancer 1989;63:2336-42.

34. Smith WB, Gracely RH, Safer MA. The meaning of pain: cancer patient's rating and recall of pain intensity and affect. Pain 1998;78:123-9.

35. Stark D, Kiely M, Smith A, Velikova G, House A, Selby P. Anxiety disorders in cancer patients: their nature, associations, and relation to quality of life. $\mathrm{J}$ Clin Oncol 2002;20:3137-48.

36. Steinman RH. The cancer patient with anxiety and chronic pain. International Association for the Study of Pain. IASP. September 2009. Volume XVII, Issue 4.

37. Levy MH, Samuel TA. Management of Cancer Pain. Seminars in Oncology 2005;32:179-93.

38. Jadad AR, Browman GP. The WHO analgesic ladder for cancer pain management: stepping up the quality of its evaluation. JAMA 1995;274:1870-3.

39. Van der Beuken J, van Everdingen MH, de Rijke JM, Kessels AG, Schouten HC, van Kleef M, et al. Prevalence of pain in patients with cancer: a systemic review of the past 40 years. Ann Oncol 2007;18: 1437-49.

40. Deng G, Cassileth BR. Integrative oncology: complementary therapies for pain, anxiety, and mood disturbance. CA Cancer J Clin 2005;55:109-16.

41. Smith WB, Gracely RH, Safer MA. The meaning of pain: cancer patient's rating and recall of pain intensity and affect. Pain 1998;78:123-9.

42. Fisch MJ, Burton AW, editors. Cancer Pain Management. New York: Mc Graw Hill; 2007.

43. Grossman SA. Cancer pain. In: Abeloff MD, et al. Abeloff's Clinical Oncology. 4th ed. Philadelphia, PA: Churchill Livingstone; 2008. p. 565.

44. Higginson IJ, Heran J, Murtagh F. Epidemiology of cancer pain. En: Sykes N, Bennett M, Yuan CS, editors. Textbook of Clinical Pain Management - cancer volume. 2nd ed. London: Hodder Arnold; 2008. p. 13-26.

45. Stjenrsward J, Gomez-Batiste X. Palliative Medicine - the global perspective: closing the know-do gap. En: Walsh D, Caraceni A, Fainsinger R, Foley $\mathrm{K}$, Glare P, Goh C, et al, editors. Palliative Medicine. Philadelphia: Elsevier; 2008. p. 2-8.

46. Bilbeny N. Medición del dolor en clínica. En: Paeile C, Saavedra A, editores. El dolor. Aspectos básicos y clínicos. Santiago: Mediterráneo; 1990. p. 87-101.

47. Bugedo G, Dagnino J, Muñoz H, Torregrosa S. Escala visual análoga: Comparación de seis escalas distintas. Rev Chil Anestesia 1989;18:132.

48. Chapman CR, Casey KL, Dubner R, Foley KM, Gracely RH, Reading AE. Pain measurement: an overview. Pain 1985;22:1-31. 
49. Huskisson EC. Measurement of pain. Lancet 1974; ii: $1127-31$.

50. Katz J, Melzack R. Measurement of pain. Anesth Clin North Am 1992;10:229-46.

51. Huskisson EC. Visual analogue scales. In: Meltzack R. Pain measurement and assessment. New York: Raven Press; 1983.p. 33-7.

52. Ohnhanus E, Adler R. Methodological problems in measurement of pain: A comparison between the verbal rating scale and the visual analogue scale. Pain 1975;1:374.

53. Portenoy RK, Miransky J, Thaler HT, Hornung J, Bianchi C, Cibas-Kong I, et al. Pain in ambulatory patients with lung or colon cancer: prevalencia, characteristics, and effect. Cancer 1992;70:1616-24.

54. Fainsinger RL, Fairchild A, NeKolaichuk C, Lawior $\mathrm{P}$, Lowe S, Hanson J. Is pain Intensity a predictor of the complexity of cancer pain management? J Clin Oncol 2009;27:585-90.

55. Appelgren L, Nordborg C, Sjöberg M, Karlsson PA, Nitescu P, Curelaru I. Spinal epidural metastasis: implications for spinal analgesia to treat "refractory" cancer pain. J Pain Symptom Manage 1997; $13: 25-42$.

56. Portenoy RK, Hagen NA. Breakthrough pain: definition, prevalence and caracteristcs. Pain 1990;41: 273-81.

57. Bruera E, Schoeller T, Wenk R, MacEachern T, Marcelino $\mathrm{S}$, Hanson J, et al. A prospective multicentre assessment of de Edmonton staging system for cancer pain. J Pain Symptom Management 1995;10: 348-55.

58. Grande LA, O’Donnell BR, Fitzgibbon DR, Terman GW. Ultra-low dose Ketamina and memantine treatment for pain in an opioid-tolerant oncology patient. Anesth Analg 2008;107:1380-3.

59. Von Moos R, Strasser F, Gillessen S, Zaugg K. Metastatic bone pain: treatment options with an emphasis on biphosphonates. Support Care Cancer 2008;16:1105-15

60. Polati E, Luzzani A, Schweiger V, Finco G, Ischia S. The role neurolytic celiac plexus block in the treatment of pancreatic cancer pain. Trasplant Proc 2008;40:1200-4.

61. Newsome S, Frawley BK, Argoff CE. Intrathecal analgesia for refractory pain. Curr Pain Headache Rep 2008;12:249-56.

62. Staats PS. Long-term intrathecal ziconotida therapy: a case study and discussion. Neuromodulation 2001;4:121-6.

63. Staas PS, Yearwood T, Charapata SG, Presley RW, Wallace MS, Byas-Smith M, et al. Intrathecal ziconotide in the treatment of refractory pain in patients with cancer or AIDS: a randomized controlled trial. JAMA 2004;291(1):63-70.

64. Uchida K. Radiofrequency treatment of the thoracic paravertebral nerve combined with glucocorticoid for refractory neuropathic pain following breast cancer surgery. Pain Physician 2009;12:277-83.

65 . Rainone F. Treating adult cancer pain in primary care. J Am Board Fam Pract 2004; 17:S48-56.

66. Collado A, Torres X, Arias A, Ruiz-López R, Mu-
ñoz-Gómez J. Tratamiento multidisciplinar en pacientes con dolor crónico en situación de baja laboral. Rev Soc Esp Dolor 2004;11: 203-9.

67. Mínguez Martí A, Monsalve Dolz V, Valia Vera JC, et al. Unidad del dolor. Integración del farmacéutico del hospital en un equipo multidisciplinar. Farm Hosp 1999;23:313-9.

68. Monsalve V, Gómez-Carretero P, Soriano J. Intervención psicológica en dolor oncológico: un estudio de revisión. Psicooncología 2006;3:139-52.

69. Brennan F, Carr DB, Cousins M. Pain management: a fundamental human right. Anesth Analg 2007;105: 205-21.

70. Portenoy RK, Payne D, Jacobsen P. Breakthrough pain: characteristics and impact in patients with cancer pain. Pain 1999;81:129-34.

71. Gómez-Batiste X. Breakthrough cancer pain: prevalence and characteristics in patients in Catalonia, Spain. J Pain Symptom Manage 2002;24:45-52.

72. Mercadante S, Radbruch L, Caraceni A, Cherny N, Kaasa S, Nauck F, et al. Episodic (breakthrough) pain: consensus conference of an expert working group of the European Association for Palliative Care. Cancer 2002;94:832-9.

73. Caraceni A, Martini C, Zecca E, Portenoy RK, Ashby MA, Hawson G, et al. Breakthrough pain characteristics and syndromes in patients with cancer pain. An international survey. Palliat Med 2004; 18:177-83.

74. Bruera E, Schoeller T, Wenk R, MacEachern T, Marcelino $\mathrm{S}$, Hanson $\mathrm{J}$, et al. A prospective multicenter assesment of the Edmonton Staging Symptom for cancer pain. J Pain Symptom Manage 1995;10:34855.

75. Maltoni M, Scarpi E, Modonesi C, Passardi A, Calpona $S$, Turriziani $A$, et al. A validation study of the WHO analgesic ladder: a two-step vs three-step strategy. Support Cancer Care 2005;13:888-94.

76. Gracia A, Trelis J, Martínez-Calderón F, Pérez-Hernández C, Sánchez-Dominguez F, Zsolt I. Citrato de fentanilo oral transmucosa en el tratamiento del dolor irruptivo en pacientes con cáncer en España. Resultados del estudio EDIPAP. Rev Soc Esp Dolor 2004;11:184-96.

77. Wlaker G, Wilcock A, Manderson C, Weller R, Crosby V. The acceptability of different routes of administration of analgesia for breakthrough pain. Palliat Med 2003;17:219-21.

78. Davies AN, Vriens J, Kennett A, McTaggart M. An observational study of oncology patients' utilization of breakthrough pain medication. J Pain Symptom Manage 2008;35:406-11.

79. William L, Macleod R. Management of breakthrough pain in patients with cancer. Drugs 2008;68: 913-24.

80. Zeppetella G. Impact and management of breakthrough pain in cancer. Curr Opin Support Palliat Care 2009;31-6.

81. Stjenrsward J, Gomez-Batiste X. Palliative Medicine - the global perspective: closing the know-do gap. En: Walsh D, Caraceni A, Fainsinger R, Foley K, Glare P, Goh C, editors. Palliative Medicine. Phi- 
ladelphia: Elsevier; 2008. p. 2-8.

82. Cassell EJ. Suffering. En: Walsh D, Caraceni A, Fainsinger R, Foley K, Glare P, Goh C, editors. Palliative Medicine. Philadelphia: Elsevier, 2008. p. 46-51.

83. Lichtental WG, Olden M, Pessin H, Breitbart W. The desire for death. En: Walsh D, Caraceni A, Fainsinger R, Foley K, Glare P, Goh C, editors. Palliative Medicine. Philadelphia: Elsevier; 2008. p. 55-9.

84. Hauser K. Clinical symptom assessment. En: Walsh D, Caraceni A, Fainsinger R, Foley K, Glare P, Goh C, editors. Palliative Medicine. Philadelphia: Elsevier; 2008. p. 325-33.

85. Glare P, Vigano A. Determining prognosis. En: Walsh D, Caraceni A, Fainsinger R, Foley K, Glare $\mathrm{P}$, Goh C, editors. Palliative Medicine. Philadelphia: Elsevier; 2008. p. 657-62.

86. Foley K. Acute and chronic cancer pain syndromes. En: Doyle D, Hanks G, Cherny N, Calman, editors. Oxford Textbook of Palliative Medicine. 3rd ed. Oxford: Oxford University Press; 2004. p. 298-315.

87. Mercadante S. Challenging pain problems. En: Walsh D, Caraceni A, Fainsinger R, Foley K, Glare P, Goh C, editors. Palliative Medicine. Philadelphia: Elsevier; 2008. p. 1425-30.

88. Portenoy RK, Forbes K, Lussier D, Hanks G. Difficult pain problems: an integrated approach. En: Walsh D, Caraceni A, Fainsinger R, Foley K, Glare P, Goh C, editors. Palliative Medicine. Philadelphia: Elsevier; 2008. p. 438-58.

89. Given B, Given CW, Sikorski A, Jean S, McCorkle $\mathrm{R}$, Champion V, et al. Establishing mild, moderate, and severe scores for cancer-related symptoms: how consistent and clinically meaningful are interference-based severity cut-points? J Pain Symptom Manage 2008;35(2):126-35.

90. Randall F. Ethical issues in cancer pain management. En: Sykes N, Bennett M, Yuan CS, editors. Textbook of Clinical Pain Management - cancer volume. 2nd ed. London: Hodder Arnold; 2008. p. 93100.

91. Cherny NI, Portenoy RK. The management of cancer pain. CA Cancer J Clin 1994;44:263-303.

92. Apolone G, Corli O, Caraceni A, Negri E, Deandrea S, Montanari M, Greco MT; Cancer Pain Outcome Research Study Group (CPOR SG) Investigators. Pattern and quality of care of cancer pain management. Results from the Cancer Pain Outcome Research Study Group. Br J Cancer 2009;100: 1566-74.

93. World Health Organization. Cancer pain relief. Geneva, 1986 (aplicación 2007: www.who.int/medicines/areas/quality_safety/delphi_study_pain_guidelines.pdf)

94. Ventafridda V, Tamburini M, Caraceni A, De Conno F, Naldi F. A validation study of the WHO method for cancer pain relief. Cancer 1987;59:850-6.

95. Levy MH. Pharmacological treatment of cancer pain. N Eng J Med 1996;10:1124-32.

96. Hassenbusch SJ, Portenoy RK. Current practices in intraspinal therapy-a survey of clinical trends and decision making. J Pain Symptom Manage 2000; 20:S4-S11.

97. Walker SM, Goudas LC, Cousins MJ, Carr DB. Combination spinal analgesic chemotherapy: a systemic review. Anesth Analg 2002;95:674-715.

98. Cormie PJ, Nairn M, Welsh J; Guideline Development Group. Control of pain in adults with cancer: summary of SIGN guidelines. BMJ 2008;337:110612.

99. Forbes K. Opioids: beliefs and myths. J Pain Palliat Care Pharmacother 2006;20:33-5.

100. Mercadante S, Villari P, Ferrera P, Casuccio A. Opioid-induced or pain relief-reduced symptoms in advanced cancer patients? Eur J Pain 2006;10:1539.

101. Pu L, Bao Gb, Xu NJ, Ma L, Pei G. Hippocampal long term potentiation is reduced by chronic opiate treatment and can be restored by re-exposure to opiates. J Neurosci 2002;22:1914-21.

102. Ho ST, Wang JJ, Huang JC, Lin MT, Liaw WJ. The magnitude of acute tolerance to morphine analgesia: concentration-dependent or time-dependent. Anesth Analg 2002;95:948-51.

103. Sees KL, Clark HW. Opioid use in the treatment of chronic pain: assessment of addiction. J Pain Symptom Manage 1993;8:257-64.

104. Schug SA, Zech D, Grond S, Jung H, Meuser T, Stobbe B. A long-term survey of morphine in cancer pain patients. J Pain Symptom Manage 1992;7:25966.

105. Højsted J, Sjøgren P. Addiction to opioids in chronic pain patients: a literature review. Eur J Pain 2007;11:490-518.

106. Cherny N, Ripamonti C, Pereira C, et al. Strategies to manage the adverse effects of oral morphine:an evidence-based report. J Clin Oncol 2001;19:254254.

107. Hanks GW, Conno F, Cherny N, Hanna M, Kalso E, McQuay HJ, et al. Morphine and alternative opioids in cancer pain: the EAPC recommendations. Br J Cancer 2001;84:587-93.

108. Mercadante S, Bruera E. Opioid switching a systematic and critical review. Cancer Treat Rev 2006;32:304-15.

109. Olsen AK, Sjogren P. Neurotoxic effects of opioids. Eur J Palliat Care 1996;3:139-42.

110. Thomas JR, von Gunten CF. Pain in terminally patients. Guidelines for Pharmacological Management. Drugs 2003;17:621-31.

111. Geppetti P, Benemei S. Pain treatment with opioids: achieving the minimal effective and the minimal interacting dose. Clin Drug Investig 2009;29(Supl.1): 3-16.

112. Steenland E, Leer JW, van Houwelingen H, Post WJ, van den Hout WB, et al. The effect of a single fraction compared to multiple fractions on painful bone metastases: a global analysis of the Dutch Bone Metastasis Study (Dutch Study). Radiother Oncol 1999;52:101-9.

113. Yarnold JR. 8 Gy single fraction radiotherapy for the treatment of metastatic skeletal pain: randomised comparison with a multifraction schedule over 12 
months of patient follow-up. Bone Pain Trial Working Party. Radiother Oncol 1999; 52:111-21.

114. Hartsell WF, Scott CB, Bruner DW, Scarantino CW, Ivker RA, Roach M 3rd, et al. Randomized trial of short- versus long-course radiotherapy for palliation of painful bone metastases. (RTOG Study). J Natl Cancer Inst 2005;97:798-804.

115. Ratanatharathorn. Metaanálisis IJRBOP: 1999;44: 1-18.

116. McQuay HJ, Collins SL, Carroll D, Moore RA. Radiotherapy for the palliation of painful bone metastases (Cochrane Review). The Cochrane Library Issue $1,2002$.

117. Rose. Recomendaciones de la A.C.R. IJRBOP. 1998; 40:117.

118. Metaanálisis. IJRBOP 2003;55:594-6.
119. Waller A, Caroline NL. Handbook of Palliative Care in Cancer. Boston: Buterworth-Heinemann; 1996.

120. Bernhard J, Dietrich D, Scheithauer W, Gerber D, Bodoky G, Ruhstaller T, et al. Clinical benefit and quality of life in patients with advanced pancreatic cancer receiving gemcitabine plus capecitabine versus gemcitabine alone: a randomized multicenter phase III clinical trial-SAKK 44/00-CECOG/PAN.1. 3.001. J Clin Oncol 2008;26:3695-701.

121. Martínez Martínez B, Casado Sáez E. Tratamientos con efecto analgésico en las metástasis óseas en dolor y cáncer. Hacia una oncología sin dolor. En: González Barón M, Ordóñez Gallego A, editores. Madrid: Editorial Médica Panamericana; 2003. p. 296-323. 